\title{
AVALIAÇÃO ELETROFISIOLÓGICA DA AUDIÇÃO EM INDIVÍDUOS APÓS TRAUMATISMO CRANIOENCEFÁLICO
}

\section{Electrophysiological hearing assessment after traumatic brain injury}

\author{
Andréa Tortosa Marangoni ${ }^{(1)}$, Renata Beatriz Fernandes Santos ${ }^{(2)}$, \\ Ítalo Capraro Suriano ${ }^{(3)}$, Karin Zazo Ortiz ${ }^{(4)}$, Daniela Gil ${ }^{(5)}$
}

\section{RESUMO}

Objetivo: caracterizar o potencial evocado auditivo de tronco encefálico e de longa latência em pacientes pós traumatismo cranioencefálico, comparando-os com indivíduos normais. Método: estudo clínico transversal realizado com 20 indivíduos audiologicamente normais divididos em dois grupos pareados por idade e sexo: a) 10 indivíduos que sofreram traumatismo cranioencefálico (grupo pesquisa); b) 10 indivíduos sem qualquer tipo de alteração neurológica (grupo controle). Foram submetidos à avaliação audiológica básica, ao potencial evocado auditivo de tronco encefálico e ao potencial evocado auditivo de longa latência (P300). Resultados: observou-se no potencial evocado auditivo de tronco encefálico, latências absolutas das ondas I, III e V e intervalo interpico I-III mais prolongados no grupo pesquisa do que no grupo controle, sendo estatisticamente significante para as latências absolutas das ondas I e III à direita e para a onda III e intervalo interpico I-III à esquerda. A amplitude das ondas I, III e V na orelha direita e das ondas III e V na orelha esquerda foram maiores no grupo controle. Em relação ao P300, o grupo pesquisa apresentou maior latência e menor amplitude em ambas as orelhas, sem significância estatística, quando comparado ao grupo controle. Conclusão: indivíduos que sofreram traumatismo cranioencefálico apresentam alterações no potencial evocado auditivo de tronco encefálico e não apresentam diferenças significantes no potencial evocado auditivo de longa latência (P300) quando comparados a indivíduos sem lesões cerebrais.

DESCRITORES: Potenciais Evocados Auditivos; Audição; Traumatismos Encefálicos

(1) Fonoaudióloga; Mestranda em Distúrbios da Comunicação Humana da Universidade Federal de São Paulo - UNIFESP; Especialização em Audiologia pela Universidade Federal de São Paulo - UNIFESP, São Paulo, Brasil.

(2) Fonoaudióloga; Mestranda em Distúrbios da Comunicação Humana da Universidade Federal de São Paulo - UNIFESP; Especialização em Distúrbios da Comunicação Humana pela Universidade Federal de São Paulo - UNIFESP, São Paulo, Brasil.

(3) Médico Neurologista; Preceptor dos residentes de Neurocirurgia da Universidade Federal de São Paulo - UNIFESP; Mestre em Neurologia pela Universidade Federal de São Paulo - UNIFESP, São Paulo, Brasil.

(4) Fonoaudióloga; Professora adjunto da Disciplina dos Distúrbios da Comunicação Humana do Departamento de Fonoaudiologia da Universidade Federal de São Paulo UNIFESP; Doutora em Ciências Humanas pela Universidade Federal de São Paulo - UNIFESP, São Paulo, Brasil.

(5) Fonoaudióloga; Professora adjunto da Disciplina dos Distúrbios da Audição do Departamento de Fonoaudiologia da Universidade Federal de São Paulo - UNIFESP; Doutora em Ciências Humanas pela Universidade Federal de São Paulo - UNIFESP, São Paulo, Brasil.

Conflito de interesses: inexistente

\section{INTRODUÇÃO}

O traumatismo cranioencefálico (TCE), condição neurológica e neurocirúrgica de grande ocorrência em nosso meio, causa danos neurológicos reversíveis ou irreversíveis, ocasionando graves e importantes seqüelas. Dentre as seqüelas que podem estar presentes estão as alterações auditivas. Estas alterações podem ser decorrentes do comprometimento da via auditiva em nível periférico - orelhas externas, média, interna e VIII nervo craniano - ou também da via auditiva em nível central' ${ }^{1}$.

Dada a freqüência com que as lesões do tronco encefálico e córtex são encontradas em pacientes com traumatismo cranioencefálico, é importante realizar os potenciais evocados auditivos de curta e longa latência para avaliar tanto a via auditiva no nível do tronco encefálico quanto as regiões corticais ${ }^{2}$. 
Os potencias evocados auditivos captam potenciais elétricos criados em vários níveis do sistema nervoso em resposta à estimulação acústica, possibilitando avaliar a integridade da via auditiva central, sendo úteis para os casos de TCE.

$\mathrm{Na}$ literatura especializada, há controversas sobre os achados no potencial evocado auditivo de tronco encefálico (PEATE) em indivíduos que sofreram TCE. Diversos estudos demonstraram alterações variadas do potencial evocado auditivo pós $\mathrm{TCE}^{3-7}$ enquanto outros não encontraram diferenças significantes ${ }^{6,8-11}$ comparando com indivíduos sem lesões cerebrais.

Ao contrário do PEATE, diversos pesquisadores verificaram que no potencial evocado auditivo de longa latência, indivíduos pós TCE apresentaram aumento da latência ${ }^{12-21}$ e diminuição da amplitude ${ }^{13-17,21-23}$ do componente P300.

Frente ao exposto, o objetivo deste estudo é caracterizar o potencial evocado auditivo de tronco encefálico (PEATE) e de longa latência (P300) em indivíduos pós-traumatismo cranioencefálico, comparando-os com indivíduos da mesma idade e sexo que não sofreram traumatismo crânioencefálico.

\section{MÉTODO}

Pesquisa transversal realizada no ambulatório de eletrofisiologia da Disciplina de Distúrbios de Audição da Universidade Federal de São Paulo (UNIFESP), durante o período de outubro de 2009 a junho de 2010.

Foram avaliados 20 indivíduos audiologicamente normais de ambos os sexos com idades entre 19 e 42 anos divididos em dois grupos pareados por idade e sexo: 10 indivíduos portadores de traumatismo cranioencefálico fechado em nível cognitivo VI, VII ou VIII da Escala de Níveis Cognitivos do Rancho Los Amigos Medical Center (grupo pesquisa - GP) e 10 indivíduos sem alterações neurológicas e/ou cognitivas evidentes (grupo controle - GC).

Todos os indivíduos foram submetidos aos seguintes procedimentos:

- Coleta da história clínica para obtenção de dados referentes à audição e ao traumatismo cranioencefálico;

- Inspeção do meato acústico externo para verificar a existência de possíveis obstruções que impossibilitariam a realização dos demais procedimentos;

- Audiometria tonal liminar e logoaudiometria (SRT - limiar de recepção de fala, IPRF - índice percentual de reconhecimento de fala), reali- zadas no audiômetro modelo GSI-61 da marca Grason-Stadler, segundo critérios adotados; ${ }^{24}$

- Medidas de imitância acústica compreendidas pela timpanometria e pela pesquisa dos reflexos acústicos no modo contralateral, obtidas no imitanciômetro AT-235 da marca Interacoustics, segundo critérios de alguns autores; ${ }^{25,26}$

- Avaliação eletrofisiológica da audição compreendida pelo PEATE e P300, realizadas no Equipamento modelo Traveler Express da marca Biologic Evoked Potencial em uma sala acusticamente tratada e protegida eletricamente, iniciadas pela captação do P300 e posteriormente pelo PEATE, já que o P300 sofre influência do estado de alerta e atenção ao estímulo acústico.

Os indivíduos foram acomodados confortavelmente em uma poltrona reclinável e foram instruídos a manter-se o mais relaxados possível, a fim de evitar artefatos miogênicos. A pele dos pacientes foi limpa com pasta abrasiva e foi aplicada pasta eletrolítica para melhor contato dos eletrodos com a pele. Os eletrodos foram fixados por meio de fita aderente e foram dispostos segundo o sistema $10-20^{27}$ da seguinte forma: eletrodo ativo (posição $C Z$ - vértex), eletrodos de referência (posição $A 1$ e A2 - lóbulos da orelha direita e esquerda, respectivamente) e eletrodo terra (localizado na fronte). A impedância dos eletrodos foi controlada de tal forma que se situasse abaixo de $5 \mathrm{k} \Omega$ e a impedância entre eletrodos se encontrasse no máximo em $2 \mathrm{k} \Omega$. Os estímulos acústicos foram apresentados por meio dos fones de inserção ER-3A, adaptados ao meato acústico externo (MAE) por meio de plugs de espuma descartáveis.

Para a obtenção do Potencial Evocado Auditivo de Longa Latência-P300 foram utilizados estímulos auditivos binaurais do tipo tone burst com freqüência de $1000 \mathrm{~Hz}$ para o estímulo freqüente e $2000 \mathrm{~Hz}$ para o estímulo raro, com intensidade de $80 \mathrm{dBNA}$ para ambos. Foram apresentados 300 estímulos por meio de um paradigma raro-freqüente, sendo a freqüência de ocorrência dos estímulos freqüentes de 240 e de 60 estímulos para os raros, ou seja, $80 \%$ para os estímulos freqüentes e $20 \%$ para os raros. A polaridade utilizada foi a alternada, a velocidade de apresentação dos estímulos ocorreu em intervalos regulares de um por segundo e o filtro utilizado foi de 0,5 a $20 \mathrm{~Hz}$. A janela utilizada foi de $750 \mathrm{~ms}$. A cada varredura, foram gravadas duas ondas, uma para o estímulo freqüente e outra para o raro, e ao final, as duas ondas foram subtraídas para a obtenção do P300. Os indivíduos foram orientados a permanecer imóveis com olhos fechados e a contar e informar o número de vezes em que o 
estímulo raro aparecesse ao final do teste. Antes de iniciar a captação do potencial, os indivíduos foram treinados para a tarefa a fim de garantir a compreensão do procedimento e para familiarizá-los com os estímulos. Para a análise deste potencial, foi considerado o valor de latência da onda P300 e os valores de normalidade utilizados foram considerados por faixa etária. ${ }^{28}$

Já para o Potencial Evocado Auditivo de Tronco Encefálico (PEATE), foram utilizados estímulos clique apresentados monoauralmente a $80 \mathrm{dBNA}$, com polaridade rarefeita e velocidade de apresentação de 19,1 cliques/segundo. Utilizou-se a janela de 0 a 10,24 milissegundos e os filtros passa baixo e passa alto foram respectivamente de $100 \mathrm{~Hz}$ e $3000 \mathrm{~Hz}$. Para analisar a reprodutibilidade do traçado, um total de 2000 estímulos foram apresentados por duas vezes. Os indivíduos foram orientados a permanecerem quietos e sem realizar movimentos com a região da cabeça e pescoço. Foram analisadas as latências absolutas das ondas I, III, e V e interpicos I-III, III-V e I-V para cada orelha e as amplitudes das ondas utilizadas foram as fornecidas pelo equipamento. Os critérios de normalidade utilizados para avaliação da integridade de via auditiva (latências absolutas e interpicos) foram os sugeridos pelo fabricante do equipamento. Foram considerados alterados os valores de latência que superaram em dois desvios padrões a normalidade sugerida.

As alterações foram classificadas em:

- Tronco encefálico baixo: quando os valores de latência das ondas III e V e, conseqüentemente os interpicos I-III e I-V encontravam-se aumentados;

- Tronco encefálico alto: quando os valores de latência da onda V e dos interpicos III-V e I-V encontravam-se aumentados;

- Alteração difusa de tronco encefálico: quando ambas as alterações supracitadas ocorreram concomitantemente na mesma orelha.

Essa pesquisa teve a aprovação do Comitê de Ética em Pesquisa da unifesp (no 1547/09). Todos os indivíduos foram informados sobre os procedimentos a serem realizados e assinaram o Termo de Consentimento Livre e Esclarecido antes de sua participação no estudo.

Após a coleta, os dados foram submetidos à análise estatística quantitativa, na qual foi utilizado o teste paramétrico ANOVA para comparar os valores por orelha das latências absolutas e amplitudes das ondas I, III, V; os valores das latências interpicos I-III, III-V e I-V do potencial evocado auditivo de tronco encefálico e valores de latência e amplitude do componente P300 do potencial evocado auditivo de longa latência entre os grupos pesquisa e controle. Os resultados com significância estatística foram destacados com asterisco $\left({ }^{*}\right)$ e os intervalos de confiança foram construídos com $95 \%$ de confiança estatística, tendo sido estabelecido nível de significância de 0,05 (5\%).

\section{RESULTADOS}

Foram avaliados 20 indivíduos divididos em dois grupos pareados por idade e sexo, 10 pertencentes ao grupo pesquisa e 10 pertencentes ao grupo controle, sendo doze do sexo masculino e oito do sexo feminino com idades variando entre 22 e 41 anos (média $=29,5$ ) e 19 e 42 anos (média $=30,25$ ) respectivamente. Os indivíduos do grupo pesquisa haviam sofrido traumatismo cranioencefálico há em média 3,1 anos e possuíam exame de imagem que evidenciava lesão focal e/ou lesão axional difusa. Vale ressaltar que dois indivíduos apresentaram alterações não especificadas no exame de tomografia computadorizada, visto que lesões microscópicas, como por exemplo, as lesões axionais difusas, não são evidenciadas neste tipo de exame, requerendo exames mais específicos, como as ressonâncias magnéticas. Infelizmente nem todos os pacientes tiveram a oportunidade de realizar este último exame, devido à dificuldade da realização em hospitais públicos e ao alto custo em serviços particulares.

$\mathrm{Na}$ análise qualitativa, observou-se que todos os indivíduos pertencentes ao grupo controle apresentaram PEATE e P300 dentro dos critérios da normalidade. Em contrapartida, das 20 orelhas analisadas dos 10 indivíduos do grupo pesquisa, 65\% apresentaram alteração no PEATE, sendo $30 \%$ da orelha direita e $35 \%$ da orelha esquerda, caracterizadas em sua maioria (50\%) por alteração de tronco encefálico baixo e 15\% por alteração difusa de tronco encefálico.

Os dados de caracterização da amostra, bem como as alterações encontradas no PEATE e no P300 quando comparado com o grupo controle estão apresentados na Figura 1. 


\begin{tabular}{|c|c|c|c|c|c|c|}
\hline Indivíduo & Idade & $\begin{array}{c}\text { Ano } \\
\text { da } \\
\text { lesão }\end{array}$ & $\begin{array}{l}\text { Exame } \\
\text { de } \\
\text { Imagem }\end{array}$ & $\begin{array}{l}\text { Impressão } \\
\text { Diagnóstica }\end{array}$ & $\begin{array}{l}\text { Alteração no } \\
\text { PEATE }\end{array}$ & $\begin{array}{l}\text { P300 comparado } \\
\text { com GC }\end{array}$ \\
\hline 1 & 27 & 2006 & TC & Não especificado & $\begin{array}{l}\text { Aumento da latência } \\
\text { absoluta da onda III } \\
\text { e do intervalo } \\
\text { interpico I-III à direita } \\
\text { e à esquerda }\end{array}$ & Aumento da latência \\
\hline 2 & 37 & 2009 & TC & $\begin{array}{l}\text { Lesão na região } \\
\text { temporal direita }\end{array}$ & $\begin{array}{c}\text { Aumento das } \\
\text { latências absoluta } \\
\text { das ondas I, III e V à } \\
\text { direita e das } \\
\text { latências absolutas } \\
\text { das ondas III, V e } \\
\text { intervalos interpicos } \\
\text { I-III e I-V à esquerda }\end{array}$ & $\begin{array}{l}\text { Aumento da latência } \\
\text { e diminuição da } \\
\text { amplitude }\end{array}$ \\
\hline 3 & 41 & 2006 & TC & $\begin{array}{c}\text { Área de } \\
\text { gliose/encefalomática } \\
\text { fronto-temporal } \\
\text { esquerda }\end{array}$ & Sem alteração & $\begin{array}{l}\text { Aumento da latência } \\
\text { e diminuição de } \\
\text { amplitude }\end{array}$ \\
\hline 4 & 32 & 2007 & $\mathrm{RM}$ & $\begin{array}{c}\text { Lesão Axional } \\
\text { Difusa/ Lesão em } \\
\text { lobo parietal e } \\
\text { occipital à esquerda }\end{array}$ & Sem alteração & $\begin{array}{c}\text { P300 sem } \\
\text { diferenciação }\end{array}$ \\
\hline 5 & 42 & 2004 & TC & $\begin{array}{l}\text { Lesões em lobo } \\
\text { frontal e temporal } \\
\text { direita/ dilatação do } \\
\text { sistema ventricular }\end{array}$ & $\begin{array}{l}\text { Aumento da latência } \\
\text { absoluta da onda III } \\
\text { e do intervalo } \\
\text { interpico } \\
\text { I-III à direita e à } \\
\text { esquerda }\end{array}$ & $\begin{array}{l}\text { Aumento da latência } \\
\text { e diminuição de } \\
\text { amplitude }\end{array}$ \\
\hline 6 & 22 & 2010 & $\mathrm{RM}$ & $\begin{array}{c}\text { Lesão Axional } \\
\text { Difusa/ Hematoma } \\
\text { frontal }\end{array}$ & $\begin{array}{l}\text { Aumento da latência } \\
\text { absoluta da onda III } \\
\text { e do intervalo } \\
\text { interpico } \\
\text { I-III à direita e à } \\
\text { esquerda }\end{array}$ & $\begin{array}{c}\text { P300 sem } \\
\text { diferenciação }\end{array}$ \\
\hline 7 & 27 & 2000 & TC & Não especificado & $\begin{array}{c}\text { Aumento dos } \\
\text { intervalos interpicos } \\
\text { I-III e I-V à esquerda }\end{array}$ & $\begin{array}{l}\text { Diminuição da } \\
\text { amplitude }\end{array}$ \\
\hline 8 & 19 & 2010 & TC & Contusão à direita & Sem alteração & $\begin{array}{l}\text { Diminuição da } \\
\text { amplitude }\end{array}$ \\
\hline 9 & 23 & 2009 & RM & $\begin{array}{c}\text { Lesão Axional } \\
\text { Difusa/ Contusão à } \\
\text { esquerda }\end{array}$ & $\begin{array}{l}\text { Aumento da latência } \\
\text { absoluta da onda III } \\
\text { e do intervalo } \\
\text { interpico } \\
\text { I-III à direita e à } \\
\text { esquerda }\end{array}$ & $\begin{array}{l}\text { Aumento da latência } \\
\text { e diminuição da } \\
\text { amplitude }\end{array}$ \\
\hline 10 & 28 & 2009 & $\mathrm{RM}$ & $\begin{array}{c}\text { Lesão Axional } \\
\text { Difusa/ Contusão à } \\
\text { direita }\end{array}$ & $\begin{array}{l}\text { Aumento do intervalo } \\
\text { interpico I-III à direita } \\
\text { e à esquerda }\end{array}$ & $\begin{array}{l}\text { Aumento da latência } \\
\text { e diminuição da } \\
\text { amplitude }\end{array}$ \\
\hline
\end{tabular}

Legenda: TC: tomografia computadorizada; RM: ressonância magnética; PEATE: potencial evocado auditivo de tronco encefálico; GC: grupo controle.

Figura 1 - Descrição do ano da lesão, dos achados dos exames de imagem e das alterações encontradas no potencial evocado auditivo de tronco encefálico e do P300 quando comparado com o grupo controle 
Já em relação ao $\mathrm{P} 300$, todos os indivíduos que sofreram TCE apresentaram latência dentro dos critérios da normalidade para faixa etária.

Quanto à análise quantitativa, observou-se que as latências absolutas das ondas I, III e V e o intervalo interpico I-III da orelha direita do PEATE foram maiores (mais prolongados) no grupo pesquisa do que no grupo controle, sendo estatisticamente significante para as latências absolutas das ondas I e III (Tabela 1).

Tabela 1 - Valores descritivos (média, mediana e desvio padrão) das latências absolutas I, III e V e intervalos interpicos I-III, III-V e I-V do potencial evocado auditivo de tronco encefálico obtidos na orelha direita no grupo pesquisa e no grupo controle

\begin{tabular}{ccccccccccc}
\hline \multicolumn{2}{c}{ Latência OD } & Média & Mediana & Desvio Padrão & CV & Min & Max & N & IC & p-valor \\
\hline \multirow{2}{*}{ I } & Pesquisa & 1,63 & 1,59 & 0,15 & $10 \%$ & 1,38 & 1,91 & 10 & 0,10 & \multirow{2}{*}{$0,018^{*}$} \\
& Controle & 1,48 & 1,50 & 0,09 & $6 \%$ & 1,32 & 1,62 & 10 & 0,06 & \\
\hline \multirow{2}{*}{ III } & Pesquisa & 3,83 & 3,80 & 0,21 & $6 \%$ & 3,51 & 4,22 & 10 & 0,13 & \multirow{2}{*}{$0,034^{*}$} \\
& Controle & 3,66 & 3,66 & 0,11 & $3 \%$ & 3,51 & 3,86 & 10 & 0,07 & \\
\hline \multirow{2}{*}{ V } & Pesquisa & 5,64 & 5,63 & 0,20 & $3 \%$ & 5,39 & 6,04 & 10 & 0,12 & \multirow{2}{*}{0,222} \\
& Controle & 5,54 & 5,57 & 0,15 & $3 \%$ & 5,22 & 5,69 & 10 & 0,09 & \\
\hline \multirow{2}{*}{ I-III } & Pesquisa & 2,20 & 2,18 & 0,16 & $7 \%$ & 1,89 & 2,36 & 10 & 0,10 & \multirow{2}{*}{0,650} \\
& Controle & 2,18 & 2,18 & 0,07 & $3 \%$ & 2,07 & 2,30 & 10 & 0,04 & \\
\hline \multirow{2}{*}{ III-V } & Pesquisa & 1,81 & 1,80 & 0,17 & $9 \%$ & 1,59 & 2,07 & 10 & 0,10 & \multirow{2}{*}{0,357} \\
& Controle & 1,88 & 1,92 & 0,18 & $9 \%$ & 1,53 & 2,07 & 10 & 0,11 & \\
\hline \multirow{2}{*}{ I-V } & Pesquisa & 4,01 & 4,04 & 0,10 & $2 \%$ & 3,84 & 4,13 & 10 & 0,06 & \multirow{2}{*}{0,480} \\
& Controle & 4,06 & 4,13 & 0,19 & $5 \%$ & 3,72 & 4,37 & 10 & 0,12 & \\
\hline
\end{tabular}

Legenda: OD: orelha direita; CV: coeficiente de variação; Min: mínimo; Max: máximo; IC: intervalo de confiança. p<0,05 ANOVA

Em relação à amplitude das ondas I, III e $\mathrm{V}$ do PEATE da mesma orelha, observou-se na Tabela 2 que a amplitude de todas as ondas foram maiores no grupo controle do que no grupo pesquisa, sem, no entanto, revelar significância estatística.

Tabela 2 - Valores descritivos (média, mediana e desvio padrão) das amplitudes das ondas I, III e V do potencial evocado auditivo de tronco encefálico obtidos na orelha direita no grupo pesquisa e no grupo controle

\begin{tabular}{ccccccc}
\hline \multirow{2}{*}{ Amplitude OD } & \multicolumn{3}{c}{ I } & \multicolumn{2}{c}{ III } & \multicolumn{2}{c}{ V } \\
\cline { 2 - 7 } & Pesquisa & Controle & Pesquisa & Controle & Pesquisa & Controle \\
\hline Média & 0,26 & 0,27 & 0,16 & 0,30 & 0,18 & 0,31 \\
Mediana & 0,27 & 0,29 & 0,12 & 0,31 & 0,11 & 0,27 \\
Desvio Padrão & 0,07 & 0,15 & 0,23 & 0,17 & 0,16 & 0,17 \\
CV & $28 \%$ & $58 \%$ & $141 \%$ & $57 \%$ & $90 \%$ & $57 \%$ \\
Min & 0,17 & 0,01 & $-0,21$ & 0,03 & 0,00 & 0,06 \\
Max & 0,36 & 0,55 & 0,63 & 0,67 & 0,53 & 0,72 \\
N & 10 & 10 & 10 & 10 & 10 & 10 \\
IC & 0,04 & 0,10 & 0,14 & 0,11 & 0,10 & 0,11 \\
\hline p-valor & \multicolumn{7}{c}{0,869} & 0,132 & \multicolumn{3}{c}{0,114} \\
\hline
\end{tabular}

Legenda: OD: orelha direita; CV: coeficiente de variação; Min: mínimo; Max: máximo; IC: intervalo de confiança. p<0,05 ANOVA 
Já para a orelha esquerda, observou-se que as latências absolutas das ondas I, III e V e o intervalo interpico I-III do PEATE foram maiores (mais prolongadas) no grupo pesquisa do que no grupo controle, sendo estatisticamente significantes a latência absoluta da onda III e o intervalo interpico I-III (Tabela 3). Em relação à amplitude, o grupo controle apresentou amplitudes das ondas III e $\mathrm{V}$ maiores do que no grupo pesquisa sem significância estatística (Tabela 4).

Tabela 3 - Valores descritivos (média, mediana e desvio padrão) das latências absolutas I, III e V e intervalos interpicos I-III, III-V e I-V do potencial evocado auditivo de tronco encefálico obtidos na orelha esquerda no grupo pesquisa e no grupo controle

\begin{tabular}{ccccccccccc}
\hline \multicolumn{2}{c}{ Latência OE } & Média & Mediana & Desvio Padrão & CV & Min & Max & N & IC & p-valor \\
\hline \multirow{2}{*}{ I } & Pesquisa & 1,54 & 1,56 & 0,18 & $11 \%$ & 1,21 & 1,74 & 10 & 0,11 & \multirow{2}{*}{0,170} \\
& Controle & 1,45 & 1,47 & 0,12 & $8 \%$ & 1,26 & 1,62 & 10 & 0,08 & \\
\hline \multirow{2}{*}{ III } & Pesquisa & 3,86 & 3,89 & 0,15 & $4 \%$ & 3,63 & 4,10 & 10 & 0,10 & \multirow{2}{*}{$0,009^{\star}$} \\
& Controle & 3,65 & 3,66 & 0,16 & $4 \%$ & 3,39 & 3,86 & 10 & 0,10 & \\
\multirow{2}{*}{ V } & Pesquisa & 5,62 & 5,60 & 0,22 & $4 \%$ & 5,34 & 5,99 & 10 & 0,14 & \multirow{2}{*}{0,335} \\
& Controle & 5,52 & 5,57 & 0,21 & $4 \%$ & 5,16 & 5,81 & 10 & 0,13 & \\
\hline \multirow{2}{*}{ I-III } & Pesquisa & 2,31 & 2,30 & 0,12 & $5 \%$ & 2,18 & 2,54 & 10 & 0,08 & \multirow{2}{*}{$0,039^{\star}$} \\
& Controle & 2,20 & 2,21 & 0,10 & $5 \%$ & 2,01 & 2,30 & 10 & 0,06 & \\
\hline \multirow{2}{*}{ III-V } & Pesquisa & 1,76 & 1,71 & 0,18 & $10 \%$ & 1,53 & 2,07 & 10 & 0,11 & \multirow{2}{*}{0,201} \\
& Controle & 1,88 & 1,89 & 0,21 & $11 \%$ & 1,42 & 2,18 & 10 & 0,13 & \\
\hline \multirow{2}{*}{ I-V } & Pesquisa & 4,08 & 4,13 & 0,17 & $4 \%$ & 3,84 & 4,31 & 10 & 0,11 & \multirow{2}{*}{1,000} \\
& Controle & 4,08 & 4,13 & 0,20 & $5 \%$ & 3,72 & 4,31 & 10 & 0,13 & \\
\hline
\end{tabular}

Legenda: OE: orelha esquerda; CV: coeficiente de variação; Min: mínimo; Max: máximo; IC: intervalo de confiança. p<0,05 ANOVA

Tabela 4 - Valores descritivos (média, mediana e desvio padrão) das amplitudes das ondas I, III e V do potencial evocado auditivo de tronco encefálico obtidos na orelha esquerda no grupo pesquisa e no grupo controle

\begin{tabular}{ccccccc}
\hline \multirow{2}{*}{ Amplitude OE } & \multicolumn{2}{c}{ I } & \multicolumn{2}{c}{ III } & \multicolumn{2}{c}{ V } \\
\cline { 2 - 7 } & Pesquisa & Controle & Pesquisa & Controle & Pesquisa & Controle \\
\hline Média & 0,32 & 0,21 & 0,14 & 0,18 & 0,21 & 0,30 \\
Mediana & 0,35 & 0,22 & 0,15 & 0,11 & 0,19 & 0,29 \\
Desvio Padrão & 0,17 & 0,12 & 0,11 & 0,20 & 0,25 & 0,07 \\
CV & $53 \%$ & $56 \%$ & $82 \%$ & $110 \%$ & $119 \%$ & $25 \%$ \\
Min & 0,03 & 0,04 & $-0,03$ & $-0,10$ & $-0,20$ & 0,19 \\
Max & 0,55 & 0,36 & 0,29 & 0,48 & 0,66 & 0,40 \\
N & 10 & 10 & 10 & 10 & 10 & 10 \\
IC & 0,10 & 0,07 & 0,07 & 0,12 & 0,15 & 0,05 \\
\hline p-valor & \multicolumn{2}{c}{0,100} & \multicolumn{3}{c}{0,576} & \multicolumn{3}{c}{0,275} \\
\hline
\end{tabular}

Legenda: OD: orelha direita; CV: coeficiente de variação; Min: mínimo; Max: máximo; IC: intervalo de confiança. p<0,05 ANOVA 
As Tabelas 5 e 6 mostram que o grupo pesquisa apresentou maior latência e menor amplitude em ambas as orelhas no potencial evocado auditivo de longa latência (P300) quando comparado ao grupo controle. Apesar das diferenças, as mesmas não foram consideradas estatisticamente significantes.

Tabela 5 - Valores descritivos (média, mediana e desvio padrão) da latência do componente P300 do potencial de longa latência obtidos nas orelhas direitas e esquerdas no grupo pesquisa e no grupo controle

\begin{tabular}{|c|c|c|c|c|}
\hline \multirow{2}{*}{ Latência (P300) } & \multicolumn{2}{|c|}{ OE } & \multicolumn{2}{|c|}{ OD } \\
\hline & Pesquisa & Controle & Pesquisa & Controle \\
\hline Média & 310,6 & 309,4 & 310,7 & 306,5 \\
\hline Mediana & 315,2 & 308,7 & 313,2 & 305,7 \\
\hline Desvio Padrão & 32,9 & 24,2 & 33,0 & 21,4 \\
\hline CV & $11 \%$ & $8 \%$ & $11 \%$ & $7 \%$ \\
\hline Min & 259,2 & 272,2 & 255,2 & 272,2 \\
\hline Max & 358,2 & 337,2 & 350,2 & 338,2 \\
\hline $\mathrm{N}$ & 10 & 10 & 10 & 10 \\
\hline IC & 20,4 & 15,0 & 20,4 & 13,3 \\
\hline
\end{tabular}

Legenda: OE: orelha esquerda; OD: orelha direita; CV: coeficiente de variação; Min: mínimo; Max: máximo; IC: intervalo de confiança. $\mathrm{p}<0,05$ ANOVA

Tabela 6 - Valores descritivos (média, mediana e desvio padrão) da amplitude do componente P300 do potencial de longa latência obtidos nas orelhas direitas e esquerdas no grupo pesquisa e no grupo controle

\begin{tabular}{|c|c|c|c|c|}
\hline \multirow{2}{*}{ Amplitude (P300) } & \multicolumn{2}{|c|}{ OE } & \multicolumn{2}{|c|}{ OD } \\
\hline & Pesquisa & Controle & Pesquisa & Controle \\
\hline Média & 6,49 & 8,08 & 6,52 & 7,08 \\
\hline Mediana & 6,47 & 8,03 & 7,78 & 7,57 \\
\hline Desvio Padrão & 2,04 & 2,44 & 2,60 & 2,69 \\
\hline CV & $31 \%$ & $30 \%$ & $40 \%$ & $38 \%$ \\
\hline Min & 3,00 & 5,03 & 1,94 & 2,92 \\
\hline Max & 9,45 & 11,72 & 9,60 & 10,70 \\
\hline $\mathrm{N}$ & 10 & 10 & 10 & 10 \\
\hline IC & 1,26 & 1,51 & 1,61 & 1,67 \\
\hline$p$-valor & \multicolumn{2}{|c|}{0,131} & \multicolumn{2}{|c|}{0,640} \\
\hline
\end{tabular}

Legenda: OE: orelha esquerda; OD: orelha direita; CV: coeficiente de variação; Min: mínimo; Max: máximo; IC: intervalo de confiança. $\mathrm{p}<0,05$ ANOVA

\section{DISCUSSÃO}

Neste tópico, será apresentada uma análise crítica dos resultados referentes ao estudo sobre a avaliação eletrofisiológica da audição (potencial evocado auditivo de tronco encefálico e potencial evocado auditivo de longa latência - P300) em indivíduos que sofreram traumatismo cranioencefálico, procurando relacioná-los com a literatura especializada. Vale ressaltar que não foram encontradas pesquisas recentes na literatura que abordasse 0 tema do estudo.

Apesar da incidência de ocorrência de traumatismo cranioencefálico ser alta, o número de indivíduos participantes deste estudo foi pequeno, visto a dificuldade de encontrar indivíduos que se 
encaixassem em todos os critérios de inclusão, principalmente com relação ao estado cognitivo.

O traumatismo cranioencefálico pode ocasionar lesões focais e/ou difusas envolvendo regiões corticais ou de tronco encefálico, devido à aceleraçãodesaceleração da cabeça que ocorre durante o acidente, gerando lesões microscópicas difusas das fibras neuronais nessas regiões, seja pelas próprias lesões focais nessas regiões, como as contusões e/ou hematomas intracranianos, ou seja pela inflamação das regiões de tronco encefálico resultantes da hipertensão intracraniana.

$\mathrm{Na}$ Figura 1 pode-se observar que as lesões dos pacientes pertencentes ao estudo foram variadas, impossibilitando a análise estatística quanto ao tipo de lesão, ou seja, não foi possível estabelecer um padrão de desempenho segundo o tipo de lesão. No entanto, é possível inferir que o traumatismo cranioencefálico, independente da lesão provocada, em sua maioria, acarreta em alterações na região de tronco encefálico, subcortical e/ou corticais, podendo ser evidenciadas através de exames auditivos eletrofisiológicos. Além disto, observa-se que independente do tempo de lesão, foram observadas alterações, demonstrando que frequentemente as lesões nessas regiões são permanentes caso o indivíduo não seja exposto a uma intervenção terapêutica específica.

As Tabelas 1 e 3 revelaram que as latências absolutas das ondas I, III e V e do intervalo interpico I-III foram mais prolongadas no grupo pesquisa do que no grupo controle, sendo estatisticamente significante para as latências absolutas das ondas I e III à direita e a latência absoluta da onda III e o intervalo interpico I-III à esquerda.

Esses achados indicam que indivíduos que sofreram traumatismo cranioencefálico apresentaram alterações na condução nervosa das vias auditivas em nível de tronco encefálico, caracterizadas principalmente por alteração de tronco encefálico baixo.

Como já citado anteriormente, as lesões do tronco encefálico e córtex cerebral são frequentes em indivíduos que sofrem traumatismo cranioencefálico. Desta forma, ao realizar um exame que avalia as regiões de tronco encefálico, é possível e justificável que se encontrem alterações, indicando a importância da realização de exames específicos que avaliem estas regiões nestes pacientes.

Diversos estudos foram realizados avaliando o potencial evocado auditivo em indivíduos que sofreram traumatismo cranioencefálico. No entanto, as pesquisas encontraram alterações variadas, sem haver consenso entre os pesquisadores, talvez pela própria variedade das lesões, mesmo que originadas por um trauma semelhante do ponto de vista do tipo de acidente e local da lesão. A seguir serão apresentados alguns achados encontrados na literatura.

Em um estudo realizado em $1977^{3}$ os pesquisadores encontraram ausência da onda III em uma das orelhas em três pacientes e intervalo interpico I-III prolongado em oito pacientes. Além disso, os autores verificaram que o intervalo interpico III-V foi mais curto em pacientes com TCE do que o normal e que o intervalo interpico I-V foi ligeiramente mais prolongado nesses indivíduos. Em outra pesquisa realizada em $1980^{4}$, observou-se que quatorze indivíduos apresentaram alteração estatisticamente significante no PEATE, com aumento das latências de um ou mais intervalos interpicos, o que corrobora com os achados do presente estudo.

Outros pesquisadores ${ }^{5}$ encontraram em seus estudos que o grupo experimental como um todo comparado com o grupo controle, apresentou latências absolutas das ondas I e III aumentadas, concordando com os achados desta pesquisa. No entanto, não foram encontradas diferenças significantes na latência absoluta da onda V, nos intervalos interpicos e na diferença interaural.

Em outro estudo ${ }^{6}$, apesar de não terem encontrado diferenças significantes entre as avaliações dos pacientes com TCE e do grupo controle, os autores relataram que o intervalo interpico I-III foi o que apresentou maior alteração.

No entanto, na literatura especializada outros autores verificaram achados diferentes quanto às latências absolutas e intervalos interpicos, como o estudo realizado em $1988^{7}$ que encontrou intervalos interpicos I-V e III-V significantemente mais prolongados em pacientes que sofreram TCE, ou que não encontraram diferenças significantes entre o grupo de pacientes que sofreram TCE e o grupo controle ${ }^{6,8-11}$, diferentemente do observado no presente estudo.

Essa variedade de achados da literatura pode ser explicada pelo fato dos estudos terem sido realizados com pacientes de diferentes graus, tipos e tempos de lesão e diferentes níveis cognitivos, o que dificulta a comparação direta dos estudos, já que estes são fatores que podem sabidamente contribuir para o aparecimento ou não de alterações.

Vale ressaltar que independente do tipo de alteração encontrada, a grande maioria dos estudos realizados encontraram alterações no potencial evocado auditivo de tronco encefálico nestes pacientes, o que mostra a importância de se realizar este procedimento em indivíduos pós TCE.

Em relação à amplitude das ondas I, III e V do potencial evocado auditivo de tronco encefálico (PEATE), observou-se nas Tabelas 2 e 4 que a amplitude de todas as ondas na orelha direita foram 
maiores no grupo controle do que no grupo pesquisa e que no grupo controle as amplitudes das ondas III e $\mathrm{V}$ foram maiores do que no grupo pesquisa na orelha esquerda, sem significância estatística.

Como o valor de amplitude não tem sido utilizado para caracterizar alterações no PEATE clinicamente, encontrou-se apenas um estudo na literatura especializada que analisou esta variável ${ }^{7}$, na qual encontrou amplitude das ondas III e V significantemente mais reduzidas em pacientes que sofreram traumatismo cranioencefálico, concordando assim com os achados encontrados nesta pesquisa. Este dado, apesar de ser pouco utilizado na prática clínica, é mais uma evidência que a região de tronco encefálico está respondendo de uma forma menos eficaz em indivíduos pós traumatismo cranioencefãlico quando comparado com indivíduos normais.

Apesar da análise qualitativa todos os indivíduos que sofreram TCE ter revelado latência absoluta do P300 dentro dos critérios de normalidade, observou-se que esses indivíduos apresentaram maior latência e menor amplitude em ambas as orelhas no potencial evocado auditivo de longa latência (P300), sem significância estatística, quando comparado com o grupo controle, corroborando com outros estudos da literatura ${ }^{12-23}$ (Tabelas 5 e 6).

Indivíduos que sofrem traumatismo cranioencefálico podem apresentar seqüelas motoras, lingüísticas, cognitivas e/ou comportamentais temporárias ou permanentes. As alterações cognitivas são frequentemente encontradas e dentre elas se encontram as alterações de memória, atenção, organização, planejamento, entre outros. O potencial evocado auditivo de longa latência - P300 é um potencial gerado por uma rede neural complexa envolvendo áreas corticais e subcorticais, e reflete a atividade eletrofisiológica cortical envolvida nas habilidades de atenção, discriminação e memória.

Como relatado anteriormente, o traumatismo cranioencefálico pode gerar lesões em regiões corticais e/ou em regiões de tronco encefálico. Visto isto, ao se utilizar um teste que avalia as regiões corticais e que envolva as habilidades de atenção e memória que frequentemente estão alteradas em sujeitos que sofreram TCE, como é o caso do potencial cognitivo P300, é explicável que estes indivíduos apresentem alterações nesta avaliação.

Com base nos resultados encontrados, apesar de não terem sido encontradas diferenças significantes no potencial evocado auditivo de longa latência (P300), pode-se comprovar a existência de alterações no potencial evocado auditivo de tronco encefálico em indivíduos que sofreram traumatismo cranioencefálico, demonstrando a utilidade da realização da avaliação eletrofisiológica da audição nesta população, de modo a contribuir para um diagnóstico audiológico mais preciso e para um planejamento mais efetivo da reabilitação destes indivíduos.

Os achados demonstraram a necessidade de realização de novas pesquisas voltadas para a área de reabilitação auditiva em indivíduos pós traumatismo cranioencefálico, podendo utilizar os potenciais evocados auditivos para monitorização terapêutica, ou seja, para monitorar as mudanças objetivas da atividade neural relacionadas com a experiência auditiva proporcionadas, como por exemplo, por meio do treinamento auditivo formal.

\section{CONCLUSÃO}

A partir da análise crítica dos resultados da avaliação eletrofisiológica de indivíduos que sofreram traumatismo cranioencefálico, conclui-se que:

- Indivíduos pós traumatismo cranioencefálico apresentam alterações de tronco encefálico baixo no potencial evocado auditivo de tronco encefálico caracterizadas por aumento das latências absolutas das ondas I e III e do intervalo interpico I-III;

- Indivíduos que sofreram traumatismo cranioencefálico não apresentam diferenças significantes no potencial evocado auditivo de longa latência (P300) quando comparados com indivíduos normais. 


\section{ABSTRACT}

Purpose: this study aimed to characterize brain stem auditory evoked potential and long latency auditory evoked potential in patients that suffered head trauma. Method: transverse clinical study performed on twenty normal hearing individuals divided in two groups paired for age and gender: a) ten individuals who suffered head trauma (trauma group); and b) ten individuals with no type of neurological alteration (control group). The individuals underwent a basic hearing evaluation as well as brain stem auditory evoked potential and long latency auditory evoked potential (P300) tests. Results: on the brain stem auditory evoked potential, more prolonged absolute latencies of Waves I, III and V and the I-III inter-peak interval were found in the trauma group in comparison with the control group, with statistically significant differences with regard to the absolute latencies of Waves I and III in the right ear and both Wave III and the I-III inter-peak interval in the left ear. The amplitude of Waves I, III and $\mathrm{V}$ in the right ear and Waves III and V in the left ear were higher in the control group. Regarding P300, the trauma group displayed greater latency and lesser amplitude in both ears comparing with the control group, with no statistical significance. Conclusion: individuals that suffered head trauma display alterations in the brain stem auditory evoked potential and do not display significant differences with regard to long latency auditory evoked potential (P300) when compared to individuals with no brain injury.

KEYWORDS: Auditory Evoked Potentials; Hearing; Brain Injuries

\section{REFERÊNCIAS}

1. Jeger $S$, Jeger J. Alterações auditivas. Um Manual para Avaliação Clínica. Rio de Janeiro: Livraria Atheneu Editora; 1989.

2. Bergemalm PO, Lyxell B. Appearances are deceptive:Long-term cognitive and central auditory sequelae from closed head injury. Int $\mathrm{J}$ Audiol. 2005;44(1):39-49.

3. Rowe MJ. The brainstem auditory evoked response in post concussion vertigo. Electroencephalogr Clin Neurophsiol. 1977;43:454 (abstract).

4. Rowe MJ, Carlson C. Brainstem auditory evoked potentials in postconcussion dizziness. Arch Neurol. 1980;37:679-83.

5. Noseworthy JH, Miller J, Murray TJ, Regan D. Auditory brainstem responses in postconcussion syndrome. Arch Neurol. 1981;38:275-8.

6. Schoenhuber R, Gentilini M, Scarano M, Bortolotti. Longitudinal study of auditory brain-stem response in patients with minor head injuries. Arch Neurol. 1987;44:1181-2.

7. Elwany S. Auditory brain stem responses (ABR) in patients with acute severe closed head injuries. $J$ Laryngol Otol. 1988;102:755-9.

8. Lindsay KW, Carlin J, Kennedy I, Fry J, Mclnnes A, Teasdale GM. Evoked potentials in severe head injury - analysis and relation to outcome. J Neurol Neurosurg Psychiatry. 1981;44:796-802.

9. Karnaze DS, Marshall LF, McCarthy CS, Klauber MR, Bickford RG. Localizing and prognostic value of auditory evoked responses in coma after closed head injury. Neurology. 1982;32:299-302.

10. Scherg M, Cramon D, Elton M. Brain-stem auditory-evoked potentials in post-comatose patients after severe head trauma. $J$ Neurol. 1984;231:1-5.

11. Schoenhuber $R$, Gentilini $M$, Orlando $A$. Prognostic value of auditory brain-stem responses for late postconcussion symptoms following minor head injury. J Neurosurg. 1988;68:742-4.

12. Olbrich HM, Nau HE, Lodemann E, Zerbin D, Schimit-Neuerburg KP. Evoked potential assessment of mental function during recovery from severe head injury. Surg Neurol. 1986; 26:112-8.

13. Rugg MD, Cowan $C P$, Nagy ME, Milner $A D$, Jacobson I, Brooks DN. Event related potentials from closed head injury patients in an auditory "oddball" task: evidence of dysfunction in stimulus categorisation. J Neurol Neurosurg Psychiatry. 1988;51:691-8.

14. Pratap-Chand R, Sinniah M, Salem FA. Cognitive evoked potential (P300): a metric for cerebral concussion. Acta Neurol Scand. 1988;78:185-9.

15. Reinvang I, Nordby H, Nielsen CS. Information processing déficits in head injury assessed with ERPs reflecting early and late processing stages. Neuropsychologia. 2000;38:995-1005.

16. Duncan $\mathrm{CC}$, Kosmidis MH, Mirsky AF. Event-related potential assessment of information processing after closed head injury. Psychophysiology. 2003;40:45-59. 
17. Lew HL, Lee EH, Pan SSL, Date ES. Electrophysiologic abnormalities of auditory and visual information processing in patients with traumatic brain injury. Am J Phys Med Rehabil. 2004;83:428-33.

18. Spikman JM, Naalt JVD, Weerden TWV, Zomeren AHV. Indices of slowness of information processing in head injury patients: test for selective attention related to ERP latencies. J Int Neuropsychol Soc. 2004;10:851-61.

19. Duncan CC, Kosmidis MH, Mirsky AF. Closed head injury-related information processing déficits: an event-related potential analysis. Int $\mathrm{J}$ Psychophysiol. 2005;58:133-57.

20. Reza MF, Ikoma K, Ito T, Ogawa T, Mano Y. N200 latency and P300 amplitude in depressed mood post-traumatic brain injury patients. Neuropsychol Rehabil. 2007;17(6):723-34.

21. Elting JW, Maurits N, Weerden T, Spikman $\mathrm{J}$, Keyser J, Naalt J. P300 analysis techniques in cognitive impairment after brain injury: comparison with neuropsychological and imaging data. Brain Inj. 2008;22(11):870-81.

22. Segalowitz SJ, Bernstein DM, Lawson S. P300 event-related potential decrements in well-functioning university students with mild head injury. Brain and Cogn. 2001;45:342-56.

23. Doi R, Morita K, Shigemori M, Tokumoti T, Maeda $\mathrm{H}$. Characteristics of cognitive function in patients after traumatic brain injury assessed by visual and auditory event-related potentials. Am J Phys Med Rehabil. 2007;86:641-9.

24. Mangabeira Albernaz $P$, Mangabeira Albernaz PL, Mangabeira Albernaz, Mangabeira Albernaz Filho P. Otorrinolaringologia Prática. São Paulo: Savier; 1981.

25. Jerger J. Clinical experience with impedance audiometry. Arch Otolaryngol. 1970;92:311-24.

26. Lopes $\mathrm{F}^{\circ} \mathrm{OC}$. Contribuição ao estudo da impedância acústica [dissertação]. São Paulo: Faculdade de Medicina da Universidade de São Paulo; 1972.

27. Jasper $\mathrm{HH}$. The ten twenty electrode system of the international federation. Electroencephalogr Clin Neurophysiol. 1958;10:371-5.

28. McPherson DL. Long Latency auditory evoked potentials. In: McPherson DL, Late Potentials of The auditory system. San Diego: Singular Publishing Group, 1996. p. 7-21.
http://dx.doi.org/10.1590/S1516-18462011005000138

RECEBIDO EM: 08/04/2011

ACEITO EM: 07/07/2011

Endereço para correspondência:

Andréa Tortosa Marangoni

Rua Nicarágua, 140 - Jardim das Nações

Taubaté - SP

CEP: $12030-500$

E-mail: andreatortosa@ hotmail.com 